\title{
3-D flow of a compressible viscous micropolar fluid with spherical symmetry: uniqueness of a generalized solution
}

\author{
Nermina Mujaković ${ }^{1}$ and Ivan Dražić 2* $^{*}$
}

"Correspondence: idrazic@riteh.hr

${ }^{2}$ Faculty of Engineering, University

of Rijeka, Rijeka, Croatia

Full list of author information is

available at the end of the article

\begin{abstract}
We consider nonstationary 3-D flow of a compressible viscous heat-conducting micropolar fluid in the domain that is the subset of $\mathbf{R}^{3}$ bounded with two concentric spheres that present the solid thermoinsulated walls. In the thermodynamical sense the fluid is perfect and polytropic. If we assume that the initial density and temperature are strictly positive and that the initial data are sufficiently smooth spherically symmetric functions then our problem has a generalized solution for a sufficiently small time interval. We study the problem in the Lagrangian description and prove the uniqueness of its generalized solution.
\end{abstract}

Keywords: micropolar fluid; spherical symmetry; generalized solution; uniqueness

\section{Introduction}

The theory of micropolar fluids was introduced by Ahmed Cemal Eringen in 1960 [1]. Eringen suggested many possible applications of the micropolar fluid, but from the mathematical point of view the theory is still in an early stage of development. The results for incompressible flow are very well systematized in the book of Lukaszewicz [2], but the theory for compressible flows, especially for flows involving temperature, is still in its beginnings.

In this paper we analyze compressible flow of isotropic, viscous, and heat-conducting micropolar fluid which is in the thermodynamical sense perfect and polytropic. The model for this type of flow was first considered by Mujaković in [3] where she developed the onedimensional model. In the same work, the local existence and uniqueness of a generalized solution for homogeneous boundary conditions were proved. In the work [4] the existence of a solution global in time for the described problem was proved. Mujaković also analyzed the regularity and stabilization for the model, as well as the Cauchy problem [5]. In her recent works, for example [6], the problem with non-homogeneous boundary condition for density, microrotation, and heat flux was analyzed.

Other authors, for example Chen et al. in [7] and [8] or Easwaran and Majumdar in [9], analyzed different kinds of problems concerning micropolar fluid, as well as in the three-dimensional case, but without the temperature. Till now the described model of compressible micropolar fluid (model with temperature) in the three-dimensional case has been considered just in [10] by Dražić and Mujaković in the spherically symmetric case.

o 2014 Mujaković and Dražić; licensee Springer. This is an Open Access article distributed under the terms of the Creative Commons Attribution License (http://creativecommons.org/licenses/by/4.0), which permits unrestricted use, distribution, and reproduction in any medium, provided the original work is properly credited. 
Spherically symmetric flow for a classical fluid was considered for example in [11-13], and [14]. Uniqueness of the solution for the problems with a classical fluid in the spherically symmetric case was proved in [15]. For a micropolar fluid in the one-dimensional case, the uniqueness results are given for example in [16] for the Cauchy problem. The uniqueness problem for the micropolar fluid was also considered in [9] but for a fluid which is not heat-conducting.

In this work we prove the uniqueness of the solution for the problem presented in [10] where we proved the local existence of generalized spherically symmetric solution for the flow of described fluid in the domain to be subset of $\mathbf{R}^{3}$ bounded with two concentric spheres that present solid thermoinsulated walls, assuming that the initial density and temperature are strictly positive and that the initial data are smooth enough spherically symmetric functions.

\section{Statement of the problem and the main result}

The motion of 3-D compressible viscous micropolar heat-conducting fluid which has the property of spherical symmetry and which is in the thermodynamical sense perfect and polytropic is described in Lagrangian coordinates by the following system of differential equations [10]:

$$
\begin{aligned}
\frac{\partial \rho}{\partial t}= & -\frac{1}{L} \rho^{2} \frac{\partial}{\partial x}\left(r^{2} v\right), \\
\frac{\partial v}{\partial t}= & -\frac{R}{L} r^{2} \frac{\partial}{\partial x}(\rho \theta)+\frac{\lambda+2 \mu}{L^{2}} r^{2} \frac{\partial}{\partial x}\left(\rho \frac{\partial}{\partial x}\left(r^{2} v\right)\right), \\
\rho \frac{\partial \omega}{\partial t}= & -\frac{4 \mu_{r}}{j_{I}} \omega+\frac{c_{0}+2 c_{d}}{j_{I} L^{2}} r^{2} \rho \frac{\partial}{\partial x}\left(\rho \frac{\partial}{\partial x}\left(r^{2} \omega\right)\right), \\
\rho \frac{\partial \theta}{\partial t}= & \frac{k}{c_{v} L^{2}} \rho \frac{\partial}{\partial x}\left(r^{4} \rho \frac{\partial \theta}{\partial x}\right)-\frac{R}{c_{v} L} \rho^{2} \theta \frac{\partial}{\partial x}\left(r^{2} v\right)+\frac{\lambda+2 \mu}{c_{v} L^{2}}\left[\rho \frac{\partial}{\partial x}\left(r^{2} v\right)\right]^{2} \\
& -\frac{4 \mu}{c_{v} L} \rho \frac{\partial}{\partial x}\left(r v^{2}\right)+\frac{c_{0}+2 c_{d}}{c_{v} L^{2}}\left[\rho \frac{\partial}{\partial x}\left(r^{2} \omega\right)\right]^{2}-\frac{4 c_{d}}{c_{v} L} \rho \frac{\partial}{\partial x}\left(r \omega^{2}\right)+\frac{4 \mu_{r}}{c_{v}} \omega^{2} .
\end{aligned}
$$

This system is considered on the set $\left.Q_{T_{0}}=\right] 0,1[\times] 0, T_{0}\left[\right.$, where $Q_{T_{0}}$ is the domain of our generalized solution (see [10]). Here $\rho, v, \omega$, and $\theta$ denote, respectively, the mass density, velocity, microrotation velocity, and temperature of the fluid. $R, L, c_{v}, j_{I}$ are positive constants. From [10] (formulas (8) and (9)) it follows that the coefficients of viscosity $\lambda, \mu$, coefficients of microviscosity $\mu_{r}, c_{0}, c_{d}$, and heat conduction coefficient $k$ have the properties:

$$
\mu, \mu_{r}, c_{d}, k \geq 0, \quad \lambda+2 \mu \geq 0, \quad c_{0}+2 c_{d} \geq 0 .
$$

Equations (1)-(4) are, respectively, local forms of the conservations laws for the mass, momentum, momentum moment, and energy.

We take the following non-homogeneous initial and boundary conditions:

$$
\begin{aligned}
& \rho(x, 0)=\rho_{0}(x), \\
& v(x, 0)=v_{0}(x), \\
& \omega(x, 0)=\omega_{0}(x),
\end{aligned}
$$




$$
\begin{aligned}
& \theta(x, 0)=\theta_{0}(x), \\
& v(0, t)=v(1, t)=0, \\
& \omega(0, t)=\omega(1, t)=0, \\
& \frac{\partial \theta}{\partial x}(0, t)=\frac{\partial \theta}{\partial x}(1, t)=0
\end{aligned}
$$

for $x \in] 0,1[, t \in] 0, T_{0}\left[\right.$. Here $\rho_{0}, v_{0}, \omega_{0}$, and $\theta_{0}$ are known real functions. We assume that there exists a constant $m \in \mathbf{R}^{+}$such that

$$
\left.\rho_{0}(x) \geq m, \quad \theta_{0}(x) \geq m \quad \text { for } x \in\right] 0,1[.
$$

The function $r$ is defined by

$$
\left.r(x, t)=r_{0}(x)+\int_{0}^{t} v(x, \tau) d \tau, \quad(x, t) \in\right] 0,1[\times] 0, T_{0}[,
$$

where

$$
\left.r_{0}(x)=\left(a^{3}+3 L \int_{0}^{x} \frac{1}{\rho_{0}(y)} d y\right)^{\frac{1}{3}}, \quad x \in\right] 0,1[
$$

$(a>0$ is radius of the smaller boundary sphere (see [10])).

In this paper we study the uniqueness of a generalized solution which is defined as follows.

Definition 2.1 A generalized solution of the problem (1)-(11) in the domain $\left.Q_{T}=\right] 0,1[\times$ ]0, $T$ [ is a quadruple of functions

$$
(x, t) \mapsto(\rho, v, \omega, \theta)(x, t), \quad(x, t) \in Q_{T},
$$

where

$$
\begin{aligned}
& \rho \in L^{\infty}\left(0, T ; H^{1}(] 0,1[)\right) \cap H^{1}\left(Q_{T}\right), \quad \inf _{Q_{T}} \rho>0, \\
& v, \omega, \theta \in L^{\infty}\left(0, T ; H^{1}(] 0,1[)\right) \cap H^{1}\left(Q_{T}\right) \cap L^{2}\left(0, T ; H^{2}(] 0,1[)\right),
\end{aligned}
$$

that satisfy equations (1)-(4) a.e. in $Q_{T}$ and conditions (5)-(11) in the sense of traces.

Remark 2.1 From the embedding and interpolation theorems (e.g. [17] and [18]) one can conclude that from (16) and (17) follows:

$$
\begin{aligned}
& \rho \in L^{\infty}(0, T ; C([0,1])) \cap C\left([0, T], L^{2}(] 0,1[)\right), \\
& v, \omega, \theta \in L^{2}\left(0, T ; C^{(1)}([0,1])\right) \cap C\left([0, T], H^{1}(] 0,1[)\right), \\
& \nu, \omega, \theta \in C\left(\bar{Q}_{T}\right) .
\end{aligned}
$$

Let the initial data (5)-(8) have the following smoothness properties:

$$
\rho_{0}, \theta_{0} \in H^{1}(] 0,1[), \quad v_{0}, \omega_{0} \in H_{0}^{1}(] 0,1[) .
$$


Remark 2.2 Because of the embedding $H^{m}(] 0,1[) \hookrightarrow C^{k}([0,1])$, for $m-k>\frac{1}{2}$ we conclude that there exists $M \in \mathbf{R}^{+}$, so that

$$
\rho_{0}(x),\left|v_{0}(x)\right|,\left|\omega_{0}(x)\right|, \theta_{0}(x) \leq M, \quad x \in[0,1]
$$

Assuming condition (21) and inequalities (12) we proved in the previous paper [10] the following local existence theorem.

Theorem 2.1 There exists small enough $T_{0} \in \mathbf{R}^{+}$such that the problem (1)-(11) has a generalized solution $(\rho, v, \omega, \theta)$ in $\left.Q_{T_{0}}=\right] 0,1[\times] 0, T_{0}[$, having the property

$$
\theta>0 \quad \text { in } \bar{Q}_{T_{0}} .
$$

For the function $r$, defined by (13) we get

$$
r \in L^{\infty}\left(0, T_{0} ; H^{2}(] 0,1[)\right) \cap H^{2}\left(Q_{T_{0}}\right) \cap C\left(\bar{Q}_{T_{0}}\right),
$$

and we easily conclude to the following property:

$$
r \in L^{\infty}\left(0, T_{0} ; C^{1}([0,1])\right)
$$

Our proof of the uniqueness does not depend on the size of the time existence interval $\left[0, T_{0}\right]$. Because of that, hereafter we will take $T_{0}=T$.

The aim of this paper is to prove the following result.

Theorem 2.2 Let the initial functions $\rho_{0}, v_{0}, \omega_{0}$, and $\theta_{0}$ satisfy conditions (12) and (21). Then the problem (1)-(11) has in the domain $Q_{T}$ at most one generalized solution $(\rho, \nu, \omega, \theta)$ with the property (23).

As has already been mentioned, the analogous theorems for the one-dimensional case have been proved in [3] and [16]. In [3] was considered the one-dimensional problem with the same type of boundary and initial conditions as in this article. In [16] there is a proof of the uniqueness theorem for the Cauchy problem of the described fluid in the onedimensional case. In the proof of Theorem 2.2 we use the method described in [19], where it has been applied for the one-dimensional case of a classical fluid (problem without the microrotation variable $\omega$ ) and also we use some ideas from [3] and [16].

\section{The proof of Theorem 2.2}

For the function $r$ defined by (13)-(14) with the properties (24)-(25) the following estimate, which we use in the proof of the theorem, holds true.

Lemma 3.1 The function $r$ satisfies the estimate

$$
r(x, t) \geq a
$$

( $a>0$ is the radius of smaller boundary sphere; see [10]) for each $(x, t) \in Q_{T}$. 
Proof We have

$$
\frac{\partial}{\partial t}\left(r^{2} \frac{\partial r}{\partial x}-\frac{L}{\rho}\right)=2 r \frac{\partial r}{\partial t} \frac{\partial r}{\partial x}+r^{2} \frac{\partial^{2} r}{\partial x \partial t}-L \frac{\partial}{\partial t}\left(\frac{1}{\rho}\right) .
$$

Taking into account (1) and (13) it follows that

$$
\frac{\partial}{\partial t}\left(r^{2} \frac{\partial r}{\partial x}-\frac{L}{\rho}\right)=2 r \frac{\partial r}{\partial x} v+r^{2} \frac{\partial v}{\partial x}-L \frac{\partial}{\partial x}\left(r^{2} v\right)=0
$$

Integrating (28) over the interval $] 0, t[$ and using (14) we get

$$
r^{2} \frac{\partial r}{\partial x}-\frac{L}{\rho}=r_{0}^{2} \frac{\partial r_{0}}{\partial x}-\frac{L}{\rho_{0}} .
$$

With the help of (16), from (29) we conclude that

$$
\frac{\partial r}{\partial x}(x, t)>0
$$

for each $(x, t) \in Q_{T}$. Therefore the function $r$ is increasing function in the variable $x$. Hence

$$
r(x, t) \geq r(0, t)=a, \quad(x, t) \in Q_{T} .
$$

Because of simplicity, hereafter we will consider the specific volume $u=\rho^{-1}$ instead of density $\rho$.

We will assume now that $\left(u_{i}, v_{i}, \omega_{i}, \theta_{i}\right), i=1,2$ are two distinct generalized solutions of the problem (1)-(11) in the domain $Q_{T}$ with the properties (12), (21), (23)-(24). Each solution is associated with a function

$$
r_{i}(x, t)=r_{0}(x)+\int_{0}^{t} v_{i}(x, \tau) d \tau, \quad i=1,2 .
$$

Now we define the functions $u=u_{1}-u_{2}, v=v_{1}-v_{2}, \omega=\omega_{1}-\omega_{2}, \theta=\theta_{1}-\theta_{2}$, and $r=r_{1}-r_{2}$. It is easy to see that the function $r$ has the property

$$
r(x, t)=\int_{0}^{t} v(x, \tau) d \tau
$$

After some calculations it can be shown that $(u, v, \omega, \theta)$ satisfy the following system:

$$
\begin{aligned}
\frac{\partial u}{\partial t}= & \frac{1}{L} \frac{\partial}{\partial x}\left(r_{1}^{2} v\right)+\frac{1}{L} \frac{\partial}{\partial x}\left(r\left(r_{1}+r_{2}\right) v_{2}\right), \\
\frac{\partial v}{\partial t}= & \alpha r_{1}^{2} \frac{\partial}{\partial x}\left[\frac{1}{u_{1}} \frac{\partial}{\partial x}\left(r_{1}^{2} v\right)+\frac{1}{u_{1}} \frac{\partial}{\partial x}\left(r\left(r_{1}+r_{2}\right) v_{2}\right)-\frac{u}{u_{1} u_{2}} \frac{\partial}{\partial x}\left(r_{2}^{2} v_{2}\right)\right] \\
& +\alpha r\left(r_{1}+r_{2}\right) \frac{\partial}{\partial x}\left[\frac{1}{u_{2}} \frac{\partial}{\partial x}\left(r_{2}^{2} v_{2}\right)\right]-\beta r_{1}^{2} \frac{\partial}{\partial x}\left[\frac{\theta}{u_{1}}-\frac{\theta_{2} u}{u_{1} u_{2}}\right] \\
& -\beta r\left(r_{1}+r_{2}\right) \frac{\partial}{\partial x}\left(\frac{\theta_{2}}{u_{2}}\right),
\end{aligned}
$$




$$
\begin{aligned}
j_{I} \frac{\partial \omega}{\partial t}= & \gamma r_{1}^{2} \frac{\partial}{\partial x}\left[\frac{1}{u_{1}} \frac{\partial}{\partial x}\left(r_{1}^{2} \omega\right)+\frac{1}{u_{1}} \frac{\partial}{\partial x}\left(r\left(r_{1}+r_{2}\right) \omega_{2}\right)-\frac{u}{u_{1} u_{2}} \frac{\partial}{\partial x}\left(r_{2}^{2} \omega_{2}\right)\right] \\
& +\gamma r\left(r_{1}+r_{2}\right) \frac{\partial}{\partial x}\left[\frac{1}{u_{2}} \frac{\partial}{\partial x}\left(r_{2}^{2} \omega_{2}\right)\right]-\delta u_{1} \omega-\delta \omega_{2} u, \\
c_{v} \frac{\partial \theta}{\partial t}= & v \frac{\partial}{\partial x}\left[\frac{r_{1}^{4}}{u_{1}} \frac{\partial \theta}{\partial x}+\frac{r\left(r_{1}+r_{2}\right)\left(r_{1}^{2}+r_{2}^{2}\right)}{u_{1}} \frac{\partial \theta_{2}}{\partial x}-\frac{u r_{2}^{4}}{u_{1} u_{2}} \frac{\partial \theta_{2}}{\partial x}\right]-\alpha \frac{u}{u_{1} u_{2}}\left[\frac{\partial}{\partial x}\left(r_{1}^{2} v_{1}\right)\right]^{2} \\
& +\frac{\alpha}{u_{2}}\left[\frac{\partial}{\partial x}\left(r_{1}^{2} v_{1}\right)+\frac{\partial}{\partial x}\left(r_{2}^{2} v_{2}\right)\right] \cdot\left[\frac{\partial}{\partial x}\left(r\left(r_{1}+r_{2}\right) v_{1}\right)+\frac{\partial}{\partial x}\left(r_{2}^{2} v\right)\right] \\
& -\frac{\beta}{u_{1}} \frac{\partial}{\partial x}\left(r_{1}^{2} v_{1}\right)\left[\theta-\frac{\theta_{2}}{u_{2}} u\right]-\beta \frac{\theta_{2}}{u_{2}}\left[\frac{\partial}{\partial x}\left(r\left(r_{1}+r_{2}\right) v_{1}\right)+\frac{\partial}{\partial x}\left(r_{2}^{2} v\right)\right] \\
& -d \frac{\partial}{\partial x}\left[r v_{1}^{2}+r_{2}\left(v_{1}+v_{2}\right) v\right]-\gamma \frac{u}{u_{1} u_{2}}\left[\frac{\partial}{\partial x}\left(r_{1}^{2} \omega_{1}\right)\right]^{2} \\
& +\frac{\gamma}{u_{2}}\left[\frac{\partial}{\partial x}\left(r_{1}^{2} \omega_{1}\right)+\frac{\partial}{\partial x}\left(r_{2}^{2} \omega_{2}\right)\right] \cdot\left[\frac{\partial}{\partial x}\left(r\left(r_{1}+r_{2}\right) \omega_{1}\right)+\frac{\partial}{\partial x}\left(r_{2}^{2} \omega\right)\right] \\
& -h \frac{\partial}{\partial x}\left[r \omega_{1}^{2}+r_{2}\left(\omega_{1}+\omega_{2}\right) \omega\right]+\delta\left[\left(\omega_{1}+\omega_{2}\right) \omega u_{1}+\omega_{2}^{2} u\right]
\end{aligned}
$$

where $\alpha=\frac{\lambda+2 \mu}{L^{2}}, \beta=\frac{R}{L}, \gamma=\frac{c_{0}+2 c_{d}}{L^{2}}, \delta=4 \mu_{r}, v=\frac{k}{L^{2}}, d=\frac{4 \mu}{L^{2}}$, and $h=\frac{4 c_{d}}{L}$.

From conditions (5)-(11) for the solutions $\left(u_{i}, v_{i}, \omega_{i}, \theta_{i}\right), i=1,2$, we can easily get the following initial and boundary conditions for the functions $u, v, \omega$, and $\theta$ :

$$
\begin{aligned}
& u(x, 0)=0, \quad v(x, 0)=0, \quad \omega(x, 0)=0, \quad \theta(x, 0)=0, \\
& v(0, t)=v(1, t)=0, \quad \omega(0, t)=\omega(1, t)=0, \\
& \frac{\partial \theta}{\partial x}(0, t)=\frac{\partial \theta}{\partial x}(1, t)=0
\end{aligned}
$$

for $x \in] 0,1[, t \in] 0, T[$. We also have $r(x, 0)=0$.

Hereafter by $C$ we denote a generic positive constant that can have different values at different places. We also use the notation

$$
\|f\|=\|f\|_{\mathrm{L}^{2}(] 0,1[)} \text {. }
$$

The proof of Theorem 2.2 is based on getting four inequalities which we use to control the bounds of the values of the functions $u, v, \omega$, and $\theta$. These inequalities we prove in the following four lemmas.

In the proofs of the lemmas we often use the following inequalities valid for the function $f$ vanishing at $x=0$ and $x=1$ or for the function with the first derivative vanishing at some point $x \in[0,1]$ :

$$
\begin{aligned}
& |f|^{2} \leq 2\|f\|\left\|\frac{\partial f}{\partial x}\right\|, \quad\|f\| \leq 2\left\|\frac{\partial f}{\partial x}\right\|, \\
& \left|\frac{\partial f}{\partial x}\right|^{2} \leq 2\left\|\frac{\partial f}{\partial x}\right\|\left\|\frac{\partial^{2} f}{\partial x^{2}}\right\|, \quad\left\|\frac{\partial f}{\partial x}\right\| \leq 2\left\|\frac{\partial^{2} f}{\partial x^{2}}\right\| .
\end{aligned}
$$

It is clear that the velocity and microrotation satisfy the inequalities (39)-(40), while the temperature satisfies the inequalities (40) only. 
Lemma 3.2 There exists a constant $C>0$ such that

$$
\|u(t)\|^{2} \leq C \int_{0}^{t}\left\|\frac{\partial v}{\partial x}(\tau)\right\|^{2} d \tau
$$

for each $t \in] 0, T[$.

Proof With the help of (13) and the Hölder inequality we can easily get

$$
\|r(t)\|^{2} \leq C \int_{0}^{t}\|v(t)\|^{2} d \tau
$$

and

$$
\left\|\frac{\partial r}{\partial x}(t)\right\|^{2} \leq C \int_{0}^{t}\left\|\frac{\partial v}{\partial x}(\tau)\right\|^{2} d \tau
$$

Multiplying (32) by $u$ and integrating over ]0,1[ we obtain the following equality:

$$
\begin{aligned}
\frac{1}{2} \frac{d}{d t}\|u(t)\|^{2}= & \frac{2}{L} \int_{0}^{1} r_{1} \frac{\partial r_{1}}{\partial x} v u d x+\frac{1}{L} \int_{0}^{1} r_{1}^{2} \frac{\partial v}{\partial x} u d x \\
& +\frac{1}{L} \int_{0}^{1} \frac{\partial r}{\partial x}\left(r_{1}+r_{2}\right) v_{2} u d x+\frac{1}{L} \int_{0}^{1} r \frac{\partial}{\partial x}\left(r_{1}+r_{2}\right) v_{2} u d x \\
& +\frac{1}{L} \int_{0}^{1} r\left(r_{1}+r_{2}\right) \frac{\partial v_{2}}{\partial x} u d x .
\end{aligned}
$$

Taking into account (25), (20), (39), (40), (42), (43), and applying the Young inequality to the integral on right-hand side in (44) we get the following estimates:

$$
\begin{aligned}
&\left|\frac{2}{L} \int_{0}^{1} r_{1} \frac{\partial r_{1}}{\partial x} v u d x\right| \leq C \max _{x \in[0,1]}\left|r_{1} \frac{\partial r_{1}}{\partial x}\right| \int_{0}^{1}|v u| d x \leq C\left(\|v(t)\|^{2}+\|u(t)\|^{2}\right) \leq C\left(\left\|\frac{\partial v}{\partial x}(t)\right\|^{2}+\|u(t)\|^{2}\right), \\
&\left|\frac{1}{L} \int_{0}^{1} r_{1}^{2} \frac{\partial v}{\partial x} u d x\right| \leq C \max _{x \in[0,1]}\left|r_{1}\right| \int_{0}^{1}\left|\frac{\partial v}{\partial x} u\right| d x \leq C\left(\left\|\frac{\partial v}{\partial x}(t)\right\|^{2}+\|u(t)\|^{2}\right), \\
&\left|\frac{1}{L} \int_{0}^{1} \frac{\partial r}{\partial x}\left(r_{1}+r_{2}\right) v_{2} u d x\right| \leq C \max _{x \in[0,1]}\left|\left(r_{1}+r_{2}\right) v_{2}\right| \int_{0}^{1}\left|\frac{\partial r}{\partial x} u\right| d x \\
& \leq C\left(\left\|\frac{\partial r}{\partial x}(t)\right\|^{2}+\|u(t)\|^{2}\right) \\
&=C\left(\int_{0}^{t}\left\|\frac{\partial v}{\partial x}(\tau)\right\|^{2} d \tau+\|u(t)\|^{2}\right), \\
&\left|\frac{1}{L} \int_{0}^{1} r \frac{\partial}{\partial x}\left(r_{1}+r_{2}\right) v_{2} u d x\right| \leq C \max _{x \in[0,1]}\left|\frac{\partial}{\partial x}\left(r_{1}+r_{2}\right) v_{2}\right| \int_{0}^{1}|r u| d x \\
& \leq C\left(\|r(t)\|^{2}+\|u(t)\|^{2}\right)=C\left(\int_{0}^{t}\|v(\tau)\|^{2} d \tau+\|u(t)\|^{2}\right) \\
& \leq C\left(\int_{0}^{t}\left\|\frac{\partial v}{\partial x}(\tau)\right\|^{2} d \tau+\|u(t)\|^{2}\right),
\end{aligned}
$$




$$
\begin{aligned}
\left|\frac{1}{L} \int_{0}^{1} r\left(r_{1}+r_{2}\right) \frac{\partial v_{2}}{\partial x} u d x\right| & \leq C \max _{x \in[0,1]}\left|r_{1}+r_{2}\right| \int_{0}^{1}\left|r \frac{\partial v_{2}}{\partial x} u\right| d x \\
& \leq C\left(\max _{x \in[0,1]}\left|\frac{\partial v_{2}}{\partial x}\right|^{2}\|u(t)\|^{2}+\|r(t)\|^{2}\right) \\
& =C\left(\max _{x \in[0,1]}\left|\frac{\partial v_{2}}{\partial x}\right|^{2}\|u(t)\|^{2}+\int_{0}^{t}\|v(\tau)\|^{2} d \tau\right) \\
& \leq C\left(\max _{x \in[0,1]}\left|\frac{\partial v_{2}}{\partial x}\right|^{2}\|u(t)\|^{2}+\int_{0}^{t}\left\|\frac{\partial v}{\partial x}(\tau)\right\|^{2} d \tau\right) \\
& \leq C\left(\left\|\frac{\partial^{2} v_{2}}{\partial x^{2}}\right\|^{2}\|u(t)\|^{2}+\int_{0}^{t}\left\|\frac{\partial v}{\partial x}(\tau)\right\|^{2} d \tau\right) .
\end{aligned}
$$

Inserting (45)-(49) into (44) and integrating over ]0, $t$ [ we get

$$
\|u(t)\|^{2} \leq C \int_{0}^{t}\left[\left(1+\left\|\frac{\partial^{2} v_{2}}{\partial x^{2}}\right\|^{2}\right)\|u(\tau)\|^{2}+\left\|\frac{\partial v}{\partial x}(\tau)\right\|^{2}\right] d \tau
$$

Using (17) for the function $v_{2}$ and applying the Gronwall inequality, from (50) follows (41).

Lemma 3.3 We have

$$
\|v(t)\|^{2}+\int_{0}^{t}\left\|\frac{\partial v}{\partial x}(\tau)\right\|^{2} d \tau \leq C \int_{0}^{t}\|\theta(\tau)\|^{2} d \tau
$$

for each $t \in] 0, T[$.

Proof In the proof of this lemma we use the procedure similar to the proof of Lemma 3.2. Multiplying (33) by $v$ and integrating over ]0,1[ we get

$$
\begin{aligned}
\frac{1}{2} \frac{d}{d t} \| & v(t) \|^{2}+\alpha \int_{0}^{1} \frac{r_{1}^{4}}{u_{1}}\left(\frac{\partial v}{\partial x}\right)^{2} d x \\
= & -4 \alpha \int_{0}^{1} \frac{r_{1}^{3}}{u_{1}} \frac{\partial r_{1}}{\partial x} v \frac{\partial v}{\partial x} d x-4 \alpha \int_{0}^{1} \frac{r_{1}^{2}}{u_{1}}\left(\frac{\partial r_{1}}{\partial x}\right)^{2} v^{2} d x \\
& -\alpha \int_{0}^{1} \frac{1}{u_{1}} \frac{\partial r}{\partial x}\left(r_{1}+r_{2}\right) v_{2} \frac{\partial}{\partial x}\left(r_{1}^{2} v\right) d x \\
& -\alpha \int_{0}^{1} \frac{1}{u_{1}} r \frac{\partial}{\partial x}\left(r_{1}+r_{2}\right) v_{2} \frac{\partial}{\partial x}\left(r_{1}^{2} v\right) d x-\alpha \int_{0}^{1} \frac{1}{u_{1}} r\left(r_{1}+r_{2}\right) \frac{\partial v_{2}}{\partial x} \frac{\partial}{\partial x}\left(r_{1}^{2} v\right) d x \\
& +\alpha \int_{0}^{1} \frac{u}{u_{1} u_{2}} \frac{\partial}{\partial x}\left(r_{2}^{2} v_{2}\right) \frac{\partial}{\partial x}\left(r_{1}^{2} v\right) d x-\alpha \int_{0}^{1} \frac{1}{u_{1}} \frac{\partial}{\partial x}\left(r_{2}^{2} v_{2}\right) \frac{\partial r}{\partial x}\left(r_{1}+r_{2}\right) v d x \\
& -\alpha \int_{0}^{1} \frac{1}{u_{1}} \frac{\partial}{\partial x}\left(r_{2}^{2} v_{2}\right) r \frac{\partial}{\partial x}\left(r_{1}+r_{2}\right) v d x-\alpha \int_{0}^{1} \frac{1}{u_{1}} \frac{\partial}{\partial x}\left(r_{2}^{2} v_{2}\right) r\left(r_{1}+r_{2}\right) \frac{\partial v}{\partial x} d x \\
& +\beta \int_{0}^{1} \frac{\theta}{u_{1}} \frac{\partial}{\partial x}\left(r_{1}^{2} v\right) d x-\beta \int_{0}^{1} \frac{\theta_{2}}{u_{1} u_{2}} u \frac{\partial}{\partial x}\left(r_{1}^{2} v\right) d x+\beta \int_{0}^{1} \frac{\theta_{2}}{u_{2}} \frac{\partial r}{\partial x}\left(r_{1}+r_{2}\right) v d x \\
& +\beta \int_{0}^{1} \frac{\theta_{2}}{u_{2}} r \frac{\partial}{\partial x}\left(r_{1}+r_{2}\right) v d x+\beta \int_{0}^{1} \frac{\theta_{2}}{u_{2}} r\left(r_{1}+r_{2}\right) \frac{\partial v}{\partial x} d x .
\end{aligned}
$$


Taking into account (18), (20), (25), (39), (40), (42), (43), the inequalities

$$
\begin{aligned}
& \left|\frac{\partial}{\partial x}\left(r_{2}^{2} v_{2}\right)\right| \leq C\left(1+\left|\frac{\partial v_{2}}{\partial x}\right|\right) \leq C\left(1+\left\|\frac{\partial^{2} v_{2}}{\partial x^{2}}\right\|\right), \\
& \left|\frac{\partial}{\partial x}\left(r_{1}^{2} v\right)\right| \leq C\left(|v|+\left|\frac{\partial v}{\partial x}\right|\right), \quad\|v\| \leq C\left\|\frac{\partial v}{\partial x}\right\|,
\end{aligned}
$$

and applying Young's inequality with a parameter $\varepsilon>0$ on the right-hand side of (52) we obtain the following inequalities:

$$
\begin{aligned}
& \left|4 \alpha \int_{0}^{1} \frac{r_{1}^{3}}{u_{1}} \frac{\partial r_{1}}{\partial x} v \frac{\partial v}{\partial x} d x\right| \leq C\|v\|\left\|\frac{\partial v}{\partial x}\right\| \leq \varepsilon\left\|\frac{\partial v}{\partial x}\right\|^{2}+C\|v\|^{2}, \\
& \left|4 \alpha \int_{0}^{1} \frac{r_{1}^{2}}{u_{1}}\left(\frac{\partial r_{1}}{\partial x}\right)^{2} v^{2} d x\right| \leq C\|v\|^{2}, \\
& \left|\alpha \int_{0}^{1} \frac{1}{u_{1}} \frac{\partial r}{\partial x}\left(r_{1}+r_{2}\right) v_{2} \frac{\partial}{\partial x}\left(r_{1}^{2} v\right) d x\right| \\
& \leq C\left(\int_{0}^{1}\left|\frac{\partial r}{\partial x}\right||v| d x+\int_{0}^{1}\left|\frac{\partial r}{\partial x}\right|\left|\frac{\partial v}{\partial x}\right| d x\right) \\
& \leq C\left(\left\|\frac{\partial r}{\partial x}\right\|^{2}+\|v\|^{2}\right)+\varepsilon\left\|\frac{\partial v}{\partial x}\right\|^{2}=\varepsilon\left\|\frac{\partial v}{\partial x}\right\|^{2}+C\left(\int_{0}^{t}\left\|\frac{\partial v}{\partial x}\right\|^{2} d \tau+\|v\|^{2}\right), \\
& \left|\alpha \int_{0}^{1} \frac{1}{u_{1}} r \frac{\partial}{\partial x}\left(r_{1}+r_{2}\right) v_{2} \frac{\partial}{\partial x}\left(r_{1}^{2} v\right) d x\right| \\
& \leq C \int_{0}^{1}|r|\left(|v|+\left|\frac{\partial v}{\partial x}\right|\right) d x \\
& \leq C\left(\|r\|^{2}+\|v\|^{2}\right)+\varepsilon\left\|\frac{\partial v}{\partial x}\right\|^{2}=\varepsilon\left\|\frac{\partial v}{\partial x}\right\|^{2}+C\left(\int_{0}^{t}\left\|\frac{\partial v}{\partial x}\right\|^{2} d \tau+\|v\|^{2}\right), \\
& \left|\alpha \int_{0}^{1} \frac{1}{u_{1}} r\left(r_{1}+r_{2}\right) \frac{\partial v_{2}}{\partial x} \frac{\partial}{\partial x}\left(r_{1}^{2} v\right) d x\right| \\
& \leq C\left\|\frac{\partial^{2} v_{2}}{\partial x^{2}}\right\| \int_{0}^{1}|r|\left(|v|+\left|\frac{\partial v}{\partial x}\right|\right) d x \\
& \leq C\left(\left\|\frac{\partial^{2} v_{2}}{\partial x^{2}}\right\|^{2}\|v\|^{2}+\|r\|^{2}\left(1+\left\|\frac{\partial^{2} v_{2}}{\partial x^{2}}\right\|^{2}\right)\right)+\varepsilon\left\|\frac{\partial v}{\partial x}\right\|^{2} \\
& \leq \varepsilon\left\|\frac{\partial v}{\partial x}\right\|^{2}+C\left(\left\|\frac{\partial^{2} v_{2}}{\partial x^{2}}\right\|^{2}\|v\|^{2}+\left(1+\left\|\frac{\partial^{2} v_{2}}{\partial x^{2}}\right\|^{2}\right) \int_{0}^{t}\left\|\frac{\partial v}{\partial x}\right\|^{2} d \tau\right), \\
& \left|\alpha \int_{0}^{1} \frac{u}{u_{1} u_{2}} \frac{\partial}{\partial x}\left(r_{2}^{2} v_{2}\right) \frac{\partial}{\partial x}\left(r_{1}^{2} v\right) d x\right| \\
& \leq C\left(1+\left\|\frac{\partial^{2} v_{2}}{\partial x^{2}}\right\|\right) \int_{0}^{1}|u|\left(|v|+\left|\frac{\partial v}{\partial x}\right|\right) d x \\
& \leq C\left(1+\left\|\frac{\partial^{2} v_{2}}{\partial x^{2}}\right\|^{2}\right)\left(\|v\|^{2}+\|u\|^{2}\right)+\varepsilon\left\|\frac{\partial v}{\partial x}\right\|^{2}, \\
& \left|\alpha \int_{0}^{1} \frac{1}{u_{1}} \frac{\partial}{\partial x}\left(r_{2}^{2} v_{2}\right) \frac{\partial r}{\partial x}\left(r_{1}+r_{2}\right) v d x\right| \\
& \leq C\left(1+\left\|\frac{\partial^{2} v_{2}}{\partial x^{2}}\right\|\right) \int_{0}^{1}|v|\left|\frac{\partial r}{\partial x}\right| d x
\end{aligned}
$$




$$
\begin{aligned}
& \left|\alpha \int_{0}^{1} \frac{1}{u_{1}} \frac{\partial}{\partial x}\left(r_{2}^{2} v_{2}\right) r \frac{\partial}{\partial x}\left(r_{1}+r_{2}\right) v d x\right| \\
& \quad \leq C\left(1+\left\|\frac{\partial^{2} v_{2}}{\partial x^{2}}\right\|\right) \int_{0}^{1}|r \| v| d x \\
& \quad \leq C\left(\left(1+\left\|\frac{\partial^{2} v_{2}}{\partial x^{2}}\right\|^{2}\right)\|v\|^{2}+\int_{0}^{t}\left\|\frac{\partial v}{\partial x}\right\|^{2} d \tau\right), \\
& \left|\alpha \int_{0}^{1} \frac{1}{u_{1}} \frac{\partial}{\partial x}\left(r_{2}^{2} v_{2}\right) r\left(r_{1}+r_{2}\right) \frac{\partial v}{\partial x} d x\right| \\
& \quad \leq C\left(1+\left\|\frac{\partial^{2} v_{2}}{\partial x^{2}}\right\|\right) \int_{0}^{1}|r|\left|\frac{\partial v}{\partial x}\right| d x \\
& \quad \leq \varepsilon\left\|\frac{\partial v}{\partial x}\right\|^{2}+C\left(1+\left\|\frac{\partial^{2} v_{2}}{\partial x^{2}}\right\|^{2}\right) \int_{0}^{t}\left\|\frac{\partial v}{\partial x}\right\|^{2} d \tau,
\end{aligned}
$$

$\left|\beta \int_{0}^{1} \frac{\theta}{u_{1}} \frac{\partial}{\partial x}\left(r_{1}^{2} v\right) d x\right| \leq C \int_{0}^{1}|\theta|\left(|v|+\left|\frac{\partial v}{\partial x}\right|\right) d x \leq C\left(\|\theta\|^{2}+\|v\|^{2}\right)+\varepsilon\left\|\frac{\partial v}{\partial x}\right\|^{2}$,

$$
\left|\beta \int_{0}^{1} \frac{\theta_{2}}{u_{1} u_{2}} u \frac{\partial}{\partial x}\left(r_{1}^{2} v\right) d x\right| \leq C \int_{0}^{1}|u|\left(|v|+\left|\frac{\partial v}{\partial x}\right|\right) d x
$$

$$
\leq C\left(\|u\|^{2}+\|v\|^{2}\right)+\varepsilon\left\|\frac{\partial v}{\partial x}\right\|^{2}
$$

$\left|\beta \int_{0}^{1} \frac{\theta_{2}}{u_{2}} \frac{\partial r}{\partial x}\left(r_{1}+r_{2}\right) v d x\right| \leq C \int_{0}^{1}\left|\frac{\partial r}{\partial x}\right||v| d x \leq C\left(\|v\|^{2}+\int_{0}^{t}\left\|\frac{\partial v}{\partial x}\right\|^{2} d \tau\right)$,

$\left|\beta \int_{0}^{1} \frac{\theta_{2}}{u_{2}} r \frac{\partial}{\partial x}\left(r_{1}+r_{2}\right) v d x\right| \leq C \int_{0}^{1}|r||v| d x \leq C\left(\|v\|^{2}+\int_{0}^{t}\left\|\frac{\partial v}{\partial x}\right\|^{2} d \tau\right)$,

$$
\left|\beta \int_{0}^{1} \frac{\theta_{2}}{u_{2}} r\left(r_{1}+r_{2}\right) \frac{\partial v}{\partial x} d x\right| \leq C \int_{0}^{1}|r|\left|\frac{\partial v}{\partial x}\right| d x \leq \varepsilon\left\|\frac{\partial v}{\partial x}\right\|^{2}+C \int_{0}^{t}\left\|\frac{\partial v}{\partial x}\right\|^{2} d \tau .
$$

Inserting (55)-(68) into (52), using (16) and (26) and integrating over ]0, $t$ [ we get

$$
\begin{aligned}
& \|v(t)\|^{2}+(1-9 \varepsilon) \int_{0}^{t}\left\|\frac{\partial v}{\partial x}\right\|^{2} d \tau \\
& \leq C \int_{0}^{t}\left[\left(1+\left\|\frac{\partial^{2} v_{2}}{\partial x^{2}}(\tau)\right\|^{2}\right)\right. \\
& \left.\quad \times\left(\|u(\tau)\|^{2}+\|v(\tau)\|^{2}+\int_{0}^{\tau}\left\|\frac{\partial v}{\partial x}(s)\right\|^{2} d s\right)+\|\theta(\tau)\|^{2}\right] d \tau .
\end{aligned}
$$

Taking into account (41) and taking $\varepsilon>0$ small enough, from (69) we immediately get

$$
\begin{aligned}
& \|v(t)\|^{2}+\int_{0}^{t}\left\|\frac{\partial v}{\partial x}\right\|^{2} d \tau \\
& \quad \leq C \int_{0}^{t}\left[\left(1+\left\|\frac{\partial^{2} v_{2}}{\partial x^{2}}(\tau)\right\|^{2}\right)\left(\|v(\tau)\|^{2}+\int_{0}^{\tau}\left\|\frac{\partial v}{\partial x}(s)\right\|^{2} d s\right)+\|\theta(\tau)\|^{2}\right] d \tau .
\end{aligned}
$$


Applying Gronwall's inequality and property (17) for the function $v_{2}$, from (70) we get

$$
\|v(t)\|^{2}+\int_{0}^{t}\left\|\frac{\partial v}{\partial x}(\tau)\right\|^{2} d \tau \leq C \int_{0}^{t}\|\theta(\tau)\|^{2} d \tau .
$$

Lemma 3.4 We have

$$
\|\omega(t)\|^{2}+\int_{0}^{t}\left\|\frac{\partial \omega}{\partial x}(\tau)\right\|^{2} d \tau \leq C \int_{0}^{t}\|\theta(\tau)\|^{2} d \tau
$$

for each $t \in] 0, T[$.

Proof As the functions $\omega_{1}, \omega_{2}$, and $\omega$ have the same properties and belong to the same spaces as the functions $v_{1}, v_{2}$, and $v$ we use the same approach as in Lemma 3.3. Multiplying (34) by $\omega$ and integrating over $] 0,1[$ we get

$$
\begin{aligned}
\frac{j_{I}}{2} \frac{d}{d t}\|\omega(t)\|^{2}+\gamma \int_{0}^{1} \frac{r_{1}^{4}}{u_{1}}\left(\frac{\partial \omega}{\partial x}\right)^{2} d x \\
=-4 \gamma \int_{0}^{1} \frac{r_{1}^{3}}{u_{1}} \frac{\partial r_{1}}{\partial x} \omega \frac{\partial \omega}{\partial x} d x-4 \gamma \int_{0}^{1} \frac{r_{1}^{2}}{u_{1}}\left(\frac{\partial r_{1}}{\partial x}\right)^{2} \omega^{2} d x \\
\quad-\gamma \int_{0}^{1} \frac{1}{u_{1}} \frac{\partial r}{\partial x}\left(r_{1}+r_{2}\right) \omega_{2} \frac{\partial}{\partial x}\left(r_{1}^{2} \omega\right) d x \\
\quad-\gamma \int_{0}^{1} \frac{1}{u_{1}} r \frac{\partial}{\partial x}\left(r_{1}+r_{2}\right) \omega_{2} \frac{\partial}{\partial x}\left(r_{1}^{2} \omega\right) d x-\gamma \int_{0}^{1} \frac{1}{u_{1}} r\left(r_{1}+r_{2}\right) \frac{\partial \omega_{2}}{\partial x} \frac{\partial}{\partial x}\left(r_{1}^{2} \omega\right) d x \\
\quad+\gamma \int_{0}^{1} \frac{u}{u_{1} u_{2}} \frac{\partial}{\partial x}\left(r_{2}^{2} \omega_{2}\right) \frac{\partial}{\partial x}\left(r_{1}^{2} \omega\right) d x-\gamma \int_{0}^{1} \frac{1}{u_{2}} \frac{\partial}{\partial x}\left(r_{2}^{2} \omega_{2}\right) \frac{\partial r}{\partial x}\left(r_{1}+r_{2}\right) \omega d x \\
\quad-\gamma \int_{0}^{1} \frac{1}{u_{2}} \frac{\partial}{\partial x}\left(r_{2}^{2} \omega_{2}\right) r \frac{\partial}{\partial x}\left(r_{1}+r_{2}\right) \omega d x-\gamma \int_{0}^{1} \frac{1}{u_{2}} \frac{\partial}{\partial x}\left(r_{2}^{2} \omega_{2}\right) r\left(r_{1}+r_{2}\right) \frac{\partial \omega}{\partial x} d x \\
\quad-\delta \int_{0}^{1} u_{1} \omega^{2} d x-\delta \int_{0}^{1} \omega_{2} u \omega d x .
\end{aligned}
$$

Now we use (18), (20), (25), (39), (40), (42), (43), (54), as well as the inequalities

$$
\begin{aligned}
& \left|\frac{\partial}{\partial x}\left(r_{2}^{2} \omega_{2}\right)\right| \leq C\left(1+\left|\frac{\partial \omega_{2}}{\partial x}\right|\right) \leq C\left(1+\left\|\frac{\partial^{2} \omega_{2}}{\partial x^{2}}\right\|\right), \\
& \left|\frac{\partial}{\partial x}\left(r_{1}^{2} \omega\right)\right| \leq C\left(|\omega|+\left|\frac{\partial \omega}{\partial x}\right|\right) .
\end{aligned}
$$

We again apply Young's inequality with a parameter $\varepsilon>0$ to the right-hand side of (73) and obtain the following estimates:

$$
\begin{aligned}
& \left|4 \gamma \int_{0}^{1} \frac{r_{1}^{2}}{u_{1}}\left(\frac{\partial r_{1}}{\partial x}\right)^{2} \omega^{2} d x\right| \leq C\|\omega\|^{2}, \\
& \left|4 \gamma \int_{0}^{1} \frac{r_{1}^{3}}{u_{1}} \frac{\partial r_{1}}{\partial x} \omega \frac{\partial \omega}{\partial x} d x\right| \leq \varepsilon\left\|\frac{\partial \omega}{\partial x}\right\|^{2}+C\|\omega\|^{2}, \\
& \left|\gamma \int_{0}^{1} \frac{1}{u_{1}} \frac{\partial r}{\partial x}\left(r_{1}+r_{2}\right) \omega_{2} \frac{\partial}{\partial x}\left(r_{1}^{2} \omega\right) d x\right|
\end{aligned}
$$




$$
\begin{aligned}
& \leq C\left(\int_{0}^{1}\left|\frac{\partial r}{\partial x}\right||\omega| d x+\int_{0}^{1}\left|\frac{\partial r}{\partial x}\right|\left|\frac{\partial \omega}{\partial x}\right| d x\right) \\
& \leq C\left(\left\|\frac{\partial r}{\partial x}\right\|^{2}+\|\omega\|^{2}\right)+\varepsilon\left\|\frac{\partial \omega}{\partial x}\right\|^{2} \\
& \leq \varepsilon\left\|\frac{\partial \omega}{\partial x}\right\|^{2}+C\left(\int_{0}^{t}\left\|\frac{\partial v}{\partial x}\right\|^{2} d \tau+\|\omega\|^{2}\right), \\
& \left|\gamma \int_{0}^{1} \frac{1}{u_{1}} r \frac{\partial}{\partial x}\left(r_{1}+r_{2}\right) \omega_{2} \frac{\partial}{\partial x}\left(r_{1}^{2} \omega\right) d x\right| \\
& \leq C \int_{0}^{1}|r|\left(|\omega|+\left|\frac{\partial \omega}{\partial x}\right|\right) d x \leq C\left(\|r\|^{2}+\|\omega\|^{2}\right)+\varepsilon\left\|\frac{\partial \omega}{\partial x}\right\|^{2} \\
& \leq \varepsilon\left\|\frac{\partial \omega}{\partial x}\right\|^{2}+C\left(\int_{0}^{t}\left\|\frac{\partial v}{\partial x}\right\|^{2} d \tau+\|\omega\|^{2}\right), \\
& \left|\gamma \int_{0}^{1} \frac{1}{u_{1}} r\left(r_{1}+r_{2}\right) \frac{\partial \omega_{2}}{\partial x} \frac{\partial}{\partial x}\left(r_{1}^{2} \omega\right) d x\right| \\
& \leq C\left\|\frac{\partial^{2} \omega_{2}}{\partial x^{2}}\right\| \int_{0}^{1}|r|\left(|\omega|+\left|\frac{\partial \omega}{\partial x}\right|\right) d x \\
& \leq \varepsilon\left\|\frac{\partial \omega}{\partial x}\right\|^{2}+C\left(\left(1+\left\|\frac{\partial^{2} \omega_{2}}{\partial x^{2}}\right\|^{2}\right) \int_{0}^{t}\left\|\frac{\partial v}{\partial x}\right\|^{2} d \tau+\|\omega\|^{2}\right), \\
& \left|\gamma \int_{0}^{1} \frac{u}{u_{1} u_{2}} \frac{\partial}{\partial x}\left(r_{2}^{2} \omega_{2}\right) \frac{\partial}{\partial x}\left(r_{1}^{2} \omega\right) d x\right| \\
& \leq C\left(1+\left\|\frac{\partial^{2} \omega_{2}}{\partial x^{2}}\right\|\right) \int_{0}^{1}|u|\left(|\omega|+\left|\frac{\partial \omega}{\partial x}\right|\right) d x \\
& \leq C\left(\left(1+\left\|\frac{\partial^{2} \omega_{2}}{\partial x^{2}}\right\|^{2}\right)\|u\|^{2}+\|\omega\|^{2}\right)+\varepsilon\left\|\frac{\partial \omega}{\partial x}\right\|^{2}, \\
& \left|\gamma \int_{0}^{1} \frac{1}{u_{2}} \frac{\partial}{\partial x}\left(r_{2}^{2} \omega_{2}\right) \frac{\partial r}{\partial x}\left(r_{1}+r_{2}\right) \omega d x\right| \\
& \leq C\left(1+\left\|\frac{\partial^{2} \omega_{2}}{\partial x^{2}}\right\|\right) \int_{0}^{1}|\omega|\left|\frac{\partial r}{\partial x}\right| d x \\
& \leq C\left[\left(1+\left\|\frac{\partial^{2} \omega_{2}}{\partial x^{2}}\right\|^{2}\right) \int_{0}^{t}\left\|\frac{\partial v}{\partial x}\right\|^{2} d \tau+\|\omega\|^{2}\right], \\
& \left|\gamma \int_{0}^{1} \frac{1}{u_{1}} \frac{\partial}{\partial x}\left(r_{2}^{2} \omega_{2}\right) r \frac{\partial}{\partial x}\left(r_{1}+r_{2}\right) \omega d x\right| \\
& \leq C\left(1+\left\|\frac{\partial^{2} \omega_{2}}{\partial x^{2}}\right\|\right) \int_{0}^{1}|r||\omega| d x \\
& \leq C\left[\left(1+\left\|\frac{\partial^{2} \omega_{2}}{\partial x^{2}}\right\|^{2}\right) \int_{0}^{t}\left\|\frac{\partial v}{\partial x}\right\|^{2} d \tau+\|\omega\|^{2}\right], \\
& \left|\gamma \int_{0}^{1} \frac{1}{u_{2}} \frac{\partial}{\partial x}\left(r_{2}^{2} \omega_{2}\right) r\left(r_{1}+r_{2}\right) \frac{\partial \omega}{\partial x} d x\right| \\
& \leq C\left(1+\left\|\frac{\partial^{2} \omega_{2}}{\partial x^{2}}\right\|\right) \int_{0}^{1}|r|\left|\frac{\partial \omega}{\partial x}\right| d x \\
& \leq \varepsilon\left\|\frac{\partial \omega}{\partial x}\right\|^{2}+C\left(1+\left\|\frac{\partial^{2} \omega_{2}}{\partial x^{2}}\right\|^{2}\right) \int_{0}^{t}\left\|\frac{\partial v}{\partial x}\right\|^{2} d \tau,
\end{aligned}
$$




$$
\begin{aligned}
& \left|\delta \int_{0}^{1} u_{1} \omega^{2} d x\right| \leq C\|\omega\|^{2}, \\
& \left|\delta \int_{0}^{1} \omega_{2} u \omega d x\right| \leq C\left(\|u\|^{2}+\|\omega\|^{2}\right) .
\end{aligned}
$$

Using the estimates (41) for the function $u$, the estimate (26) for the function $r_{1}$ and the property (16) for the function $u_{1}^{-1}$, after inserting (76)-(86) into (73) and integrating over ] $0, t[$ we get

$$
\begin{aligned}
& \|\omega(t)\|^{2}+(1-6 \varepsilon) \int_{0}^{t}\left\|\frac{\partial \omega}{\partial x}\right\|^{2} d \tau \\
& \leq C \int_{0}^{t}\left[\|\omega(\tau)\|^{2}+\int_{0}^{\tau}\left\|\frac{\partial \omega}{\partial x}(s)\right\|^{2} d s+\left(1+\left\|\frac{\partial^{2} \omega_{2}}{\partial x^{2}}(\tau)\right\|^{2}\right) \int_{0}^{\tau}\left\|\frac{\partial v}{\partial x}(s)\right\|^{2} d s\right] d \tau .
\end{aligned}
$$

We take $\varepsilon$ small enough. Applying Gronwall's inequality to (87) we get

$$
\begin{aligned}
\|\omega(t)\|^{2}+\int_{0}^{t}\left\|\frac{\partial \omega}{\partial x}\right\|^{2} d \tau & \leq C \int_{0}^{t}\left(1+\left\|\frac{\partial^{2} \omega_{2}}{\partial x^{2}}(\tau)\right\|^{2}\right) \int_{0}^{\tau}\left\|\frac{\partial v}{\partial x}(s)\right\|^{2} d s d \tau \\
& \leq C \int_{0}^{t}\left\|\frac{\partial v}{\partial x}(s)\right\|^{2} d s \int_{0}^{t}\left(1+\left\|\frac{\partial^{2} \omega_{2}}{\partial x^{2}}(\tau)\right\|^{2}\right) d \tau .
\end{aligned}
$$

Now, using the inclusion (17) for the function $\omega_{2}$, from (88) we conclude that

$$
\|\omega(t)\|^{2}+\int_{0}^{t}\left\|\frac{\partial \omega}{\partial x}(\tau)\right\|^{2} d \tau \leq C \int_{0}^{t}\left\|\frac{\partial v}{\partial x}(\tau)\right\|^{2} d \tau
$$

for each $t \in] 0, T$. Applying the inequality (51) to (89) we immediately get (72).

Lemma 3.5 We have

$$
\|\theta(t)\|^{2}+\int_{0}^{t}\left\|\frac{\partial \theta}{\partial x}(\tau)\right\|^{2} d \tau \leq C \int_{0}^{t}\|\theta(\tau)\|^{2} d \tau
$$

for each $t \in] 0, T[$.

Proof Multiplying (35) by $\theta$, using (38) and (11), after integrating by parts over ]0,1[ we get

$$
\begin{aligned}
\frac{c_{v}}{2} \frac{d}{d t}\|\theta(t)\|^{2}+v \int_{0}^{1} \frac{r_{1}^{2}}{u_{1}}\left(\frac{\partial \theta}{\partial x}\right)^{2} d x \\
=-v \int_{0}^{1} \frac{r\left(r_{1}+r_{2}\right)\left(r_{1}^{2}+r_{2}^{2}\right)}{u_{1}} \frac{\partial \theta_{2}}{\partial x} \frac{\partial \theta}{\partial x} d x+v \int_{0}^{1} \frac{u}{u_{1} u_{2}} r_{2}^{4} \frac{\partial \theta_{2}}{\partial x} \frac{\partial \theta}{\partial x} d x \\
\quad-\alpha \int_{0}^{1} \frac{u}{u_{1} u_{2}}\left[\frac{\partial}{\partial x}\left(r_{1}^{2} v_{1}\right)\right]^{2} \theta d x \\
\quad+\alpha \int_{0}^{1} \frac{1}{u_{2}}\left[\frac{\partial}{\partial x}\left(r_{1}^{2} v_{1}\right)+\frac{\partial}{\partial x}\left(r_{2}^{2} v_{2}\right)\right] \frac{\partial}{\partial x}\left(r\left(r_{1}+r_{2}\right) v_{1}\right) \theta d x \\
\quad+\alpha \int_{0}^{1} \frac{1}{u_{2}}\left[\frac{\partial}{\partial x}\left(r_{1}^{2} v_{1}\right)+\frac{\partial}{\partial x}\left(r_{2}^{2} v_{2}\right)\right] \frac{\partial}{\partial x}\left(r_{2}^{2} v\right) \theta d x
\end{aligned}
$$




$$
\begin{aligned}
& -\beta \int_{0}^{1} \frac{1}{u_{1}} \frac{\partial}{\partial x}\left(r_{1}^{2} v_{1}\right) \theta^{2} d x+\beta \int_{0}^{1} \frac{1}{u_{1}} \frac{\partial}{\partial x}\left(r_{1}^{2} v_{1}\right) \frac{\theta_{2}}{u_{2}} u \theta d x \\
& -\beta \int_{0}^{1} \frac{\theta_{2}}{u_{2}} \frac{\partial}{\partial x}\left(r\left(r_{1}+r_{2}\right) v_{1}\right) \theta d x-\beta \int_{0}^{1} \frac{\theta_{2}}{u_{2}} \frac{\partial}{\partial x}\left(r_{2}^{2} v\right) \theta d x \\
& +d \int_{0}^{1}\left(r v_{1}^{2}+r_{2}\left(v_{1}+v_{2}\right) v\right) \frac{\partial \theta}{\partial x} d x-\gamma \int_{0}^{1} \frac{u}{u_{1} u_{2}}\left[\frac{\partial}{\partial x}\left(r_{1}^{2} \omega_{1}\right)\right]^{2} \theta d x \\
& +\gamma \int_{0}^{1} \frac{1}{u_{2}}\left[\frac{\partial}{\partial x}\left(r_{1}^{2} \omega_{1}\right)+\frac{\partial}{\partial x}\left(r_{2}^{2} \omega_{2}\right)\right] \frac{\partial}{\partial x}\left(r\left(r_{1}+r_{2}\right) \omega_{1}\right) \theta d x \\
& +\gamma \int_{0}^{1} \frac{1}{u_{2}}\left[\frac{\partial}{\partial x}\left(r_{1}^{2} \omega_{1}\right)+\frac{\partial}{\partial x}\left(r_{2}^{2} \omega_{2}\right)\right] \frac{\partial}{\partial x}\left(r_{2}^{2} \omega\right) \theta d x+h \int_{0}^{1} r \omega_{1}^{2} \frac{\partial \theta}{\partial x} d x \\
& +h \int_{0}^{1} r_{2} \omega\left(\omega_{1}+\omega_{2}\right) \frac{\partial \theta}{\partial x} d x+\delta \int_{0}^{1}\left(\omega_{1}+\omega_{2}\right) u_{1} \omega \theta d x+\delta \int_{0}^{1} \omega_{2}^{2} u \theta d x
\end{aligned}
$$

To get the estimates of the integrals on the right-hand side in (91) we use again the properties (18), (20), (25), (39), (40), (42), (43), (53) for the functions $r_{1}^{2} v_{1}, r_{2}^{2} v_{2}, r_{1}^{2} \omega_{1}$, and $r_{2}^{2} \omega_{2}$, as well as the property (54) for the functions $v$ and $\omega$. We also use the inequality

$$
\left|\frac{\partial \theta_{2}}{\partial x}\right| \leq C\left\|\frac{\partial^{2} \theta_{2}}{\partial x^{2}}\right\|
$$

and the result of Lemma 3.2. As we use the same procedure as in previous proofs, we omit the details and write the final form of the estimates only. We get the following inequalities:

$$
\begin{aligned}
& \left|v \int_{0}^{1} \frac{r\left(r_{1}+r_{2}\right)\left(r_{1}^{2}+r_{2}^{2}\right)}{u_{1}} \frac{\partial \theta_{2}}{\partial x} \frac{\partial \theta}{\partial x} d x\right| \leq \varepsilon\left\|\frac{\partial \theta}{\partial x}\right\|^{2}+C\left\|\frac{\partial^{2} \theta_{2}}{\partial x^{2}}\right\|^{2} \int_{0}^{t}\left\|\frac{\partial v}{\partial x}\right\|^{2} d \tau \\
& \left|v \int_{0}^{1} \frac{u}{u_{1} u_{2}} r_{2}^{4} \frac{\partial \theta_{2}}{\partial x} \frac{\partial \theta}{\partial x} d x\right| \leq \varepsilon\left\|\frac{\partial \theta}{\partial x}\right\|^{2}+C\left\|\frac{\partial^{2} \theta_{2}}{\partial x^{2}}\right\|^{2}\|u\|^{2} \\
& \leq \varepsilon\left\|\frac{\partial \theta}{\partial x}\right\|^{2}+C\left\|\frac{\partial^{2} \theta_{2}}{\partial x^{2}}\right\|^{2} \int_{0}^{t}\left\|\frac{\partial v}{\partial x}\right\|^{2} d \tau, \\
& \left|\alpha \int_{0}^{1} \frac{u}{u_{1} u_{2}}\left[\frac{\partial}{\partial x}\left(r_{1}^{2} v_{1}\right)\right]^{2} \theta d x\right| \leq C\left(1+\left\|\frac{\partial^{2} v_{1}}{\partial x^{2}}\right\|^{2}\right)\left(\|u\|^{2}+\|\theta\|^{2}\right) \\
& \leq C\left(1+\left\|\frac{\partial^{2} v_{1}}{\partial x^{2}}\right\|^{2}\right)\left(\int_{0}^{t}\left\|\frac{\partial v}{\partial x}\right\|^{2} d \tau+\|\theta\|^{2}\right), \\
& \left|\alpha \int_{0}^{1} \frac{1}{u_{2}}\left[\frac{\partial}{\partial x}\left(r_{1}^{2} v_{1}\right)+\frac{\partial}{\partial x}\left(r_{2}^{2} v_{2}\right)\right] \frac{\partial}{\partial x}\left(r\left(r_{1}+r_{2}\right) v_{1}\right) \theta d x\right| \\
& \leq C\left(1+\left\|\frac{\partial^{2} v_{1}}{\partial x^{2}}\right\|^{2}+\left\|\frac{\partial^{2} v_{2}}{\partial x^{2}}\right\|^{2}\right)\left(\int_{0}^{t}\left\|\frac{\partial v}{\partial x}\right\|^{2} d \tau+\|\theta\|^{2}\right), \\
& \left|\alpha \int_{0}^{1} \frac{1}{u_{2}}\left[\frac{\partial}{\partial x}\left(r_{1}^{2} v_{1}\right)+\frac{\partial}{\partial x}\left(r_{2}^{2} v_{2}\right)\right] \frac{\partial}{\partial x}\left(r_{2}^{2} v\right) \theta d x\right| \\
& \leq C\left(1+\left\|\frac{\partial^{2} v_{1}}{\partial x^{2}}\right\|^{2}+\left\|\frac{\partial^{2} v_{2}}{\partial x^{2}}\right\|^{2}\right)\|\theta\|^{2}+C\left(\|v\|^{2}+\left\|\frac{\partial v}{\partial x}\right\|^{2}\right) \\
& \leq C\left(1+\left\|\frac{\partial^{2} v_{1}}{\partial x^{2}}\right\|^{2}+\left\|\frac{\partial^{2} v_{2}}{\partial x^{2}}\right\|^{2}\right)\|\theta\|^{2}+C\left\|\frac{\partial v}{\partial x}\right\|^{2},
\end{aligned}
$$


$\left|\beta \int_{0}^{1} \frac{\theta_{2}}{u_{2}} \frac{\partial}{\partial x}\left(r_{2}^{2} v\right) \theta d x\right| \leq C\left(\|\theta\|^{2}+\|v\|^{2}+\left\|\frac{\partial v}{\partial x}\right\|^{2}\right) \leq C\left(\|\theta\|^{2}+\left\|\frac{\partial v}{\partial x}\right\|^{2}\right)$,

$\left|d \int_{0}^{1}\left(r v_{1}^{2}+r_{2}\left(v_{1}+v_{2}\right) v\right) \frac{\partial \theta}{\partial x} d x\right| \leq \varepsilon\left\|\frac{\partial \theta}{\partial x}\right\|^{2}+C\left(\|v\|^{2}+\int_{0}^{t}\left\|\frac{\partial v}{\partial x}\right\|^{2} d \tau\right)$ $\leq \varepsilon\left\|\frac{\partial \theta}{\partial x}\right\|^{2}+C\left(\left\|\frac{\partial v}{\partial x}\right\|^{2}+\int_{0}^{t}\left\|\frac{\partial v}{\partial x}\right\|^{2} d \tau\right)$

$\left|\gamma \int_{0}^{1} \frac{u}{u_{1} u_{2}}\left[\frac{\partial}{\partial x}\left(r_{1}^{2} \omega_{1}\right)\right]^{2} \theta d x\right| \leq C\left(1+\left\|\frac{\partial^{2} \omega_{1}}{\partial x^{2}}\right\|^{2}\right)\left(\|u\|^{2}+\|\theta\|^{2}\right)$ $\leq C\left(1+\left\|\frac{\partial^{2} \omega_{1}}{\partial x^{2}}\right\|^{2}\right)\left(\int_{0}^{t}\left\|\frac{\partial v}{\partial x}\right\|^{2} d \tau+\|\theta\|^{2}\right)$,

$\left|\gamma \int_{0}^{1} \frac{1}{u_{2}}\left[\frac{\partial}{\partial x}\left(r_{1}^{2} \omega_{1}\right)+\frac{\partial}{\partial x}\left(r_{2}^{2} \omega_{2}\right)\right] \frac{\partial}{\partial x}\left(r\left(r_{1}+r_{2}\right) \omega_{1}\right) \theta d x\right|$

$$
\leq C\left(1+\left\|\frac{\partial^{2} \omega_{1}}{\partial x^{2}}\right\|^{2}+\left\|\frac{\partial^{2} \omega_{2}}{\partial x^{2}}\right\|^{2}\right)\left(\int_{0}^{t}\left\|\frac{\partial v}{\partial x}\right\|^{2} d \tau+\|\theta\|^{2}\right)
$$

$\left|\gamma\left[\frac{\partial}{\partial x}\left(r_{1}^{2} \omega_{1}\right)+\frac{\partial}{\partial x}\left(r_{2}^{2} \omega_{2}\right)\right] \frac{\partial}{\partial x}\left(r_{2}^{2} \omega\right) \theta d x\right|$

$$
\leq C\left(1+\left\|\frac{\partial^{2} \omega_{1}}{\partial x^{2}}\right\|^{2}+\left\|\frac{\partial^{2} \omega_{2}}{\partial x^{2}}\right\|^{2}\right)\|\theta\|^{2}+C\left(\left\|\frac{\partial \omega}{\partial x}\right\|^{2}+\|\omega\|^{2}\right)
$$$$
\leq C\left(1+\left\|\frac{\partial^{2} \omega_{1}}{\partial x^{2}}\right\|^{2}+\left\|\frac{\partial^{2} \omega_{2}}{\partial x^{2}}\right\|^{2}\right)\|\theta\|^{2}+C\left\|\frac{\partial \omega}{\partial x}\right\|^{2},
$$

$\left|h \int_{0}^{1} r \omega_{1}^{2} \frac{\partial \theta}{\partial x} d x\right| \leq \varepsilon\left\|\frac{\partial \theta}{\partial x}\right\|^{2}+C \int_{0}^{t}\left\|\frac{\partial v}{\partial x}\right\|^{2} d \tau$,

$\left|h \int_{0}^{1} r_{2} \omega\left(\omega_{1}+\omega_{2}\right) \frac{\partial \theta}{\partial x} d x\right| \leq \varepsilon\left\|\frac{\partial \theta}{\partial x}\right\|^{2}+C\|\omega\|^{2} \leq \varepsilon\left\|\frac{\partial \theta}{\partial x}\right\|^{2}+C\left\|\frac{\partial \omega}{\partial x}\right\|^{2}$,

$\left|\delta \int_{0}^{1}\left(\omega_{1}+\omega_{2}\right) u_{1} \omega \theta d x\right| \leq C\left(\|\omega\|^{2}+\|\theta\|^{2}\right) \leq C\left(\left\|\frac{\partial \omega}{\partial x}\right\|^{2}+\|\theta\|^{2}\right)$,

$\left|\delta \int_{0}^{1} \omega_{2}^{2} u \theta d x\right| \leq C\left(\|u\|^{2}+\|\theta\|^{2}\right) \leq C\left(\int_{0}^{t}\left\|\frac{\partial v}{\partial x}\right\|^{2} d \tau+\|\theta\|^{2}\right)$.

Now we will again apply (26) as well as (16). By inserting (93)-(109) into (91) and integrating over $] 0, t[$ we get

$$
\begin{aligned}
\|\theta\|^{2} & +(1-5 \varepsilon) \int_{0}^{t}\left\|\frac{\partial \theta}{\partial x}(\tau)\right\|^{2} d \tau \\
\leq & C \int_{0}^{t} A(\tau)\left(\int_{0}^{\tau}\left\|\frac{\partial v}{\partial x}(s)\right\|^{2}+\|\theta(s)\|^{2}\right) d \tau \\
& +C\left(\int_{0}^{t}\left\|\frac{\partial \omega}{\partial x}(\tau)\right\|\left\|^{2} d \tau+\int_{0}^{t}\right\| \frac{\partial v}{\partial x}(\tau) \|^{2} d \tau\right),
\end{aligned}
$$


where

$$
\begin{aligned}
A(\tau)= & 1+\left\|\frac{\partial^{2} \theta_{2}}{\partial x^{2}}(\tau)\right\|^{2}+\left\|\frac{\partial^{2} v_{1}}{\partial x^{2}}(\tau)\right\|^{2}+\left\|\frac{\partial^{2} v_{2}}{\partial x^{2}}(\tau)\right\|^{2} \\
& +\left\|\frac{\partial^{2} \omega_{1}}{\partial x^{2}}(\tau)\right\|^{2}+\left\|\frac{\partial^{2} \omega_{2}}{\partial x^{2}}(\tau)\right\|^{2} \cdot
\end{aligned}
$$

If we choose $\varepsilon$ small enough, using the properties (72), (51), and (17) we get

$$
\begin{aligned}
& \|\theta\|^{2}+\int_{0}^{t}\left\|\frac{\partial \theta}{\partial x}(\tau)\right\|^{2} d \tau \\
& \quad \leq C \int_{0}^{t} A(\tau)\left(\|\theta(\tau)\|^{2}+\int_{0}^{\tau}\left\|\frac{\partial \theta}{\partial x}(s)\right\|^{2}\right) d \tau+C \int_{0}^{t}\|\theta(\tau)\|^{2} d \tau .
\end{aligned}
$$

Applying Gronwall's inequality to (112) we finally get

$$
\|\theta(t)\|^{2}+\int_{0}^{t}\left\|\frac{\partial \theta}{\partial x}(\tau)\right\|^{2} d \tau \leq C \int_{0}^{t}\|\theta(\tau)\|^{2} d \tau
$$

Now we can easily conclude that Theorem 2.2 is valid. If we apply Gronwall's inequality again to (113) we immediately get

$$
\theta=0 .
$$

Inserting (114) into (51), and (72) we can conclude that

$$
v=0, \quad \omega=0 .
$$

Finally, from (41) we get

$$
u=0
$$

and Theorem 2.2 is proved.

\section{Competing interests}

The authors declare that they have no competing interests.

Authors' contributions

The paper is the result of joint work of both authors, who contributed equally to the final version of the paper. All authors read and approved the final manuscript.

Author details

${ }^{1}$ Department of Mathematics, University of Rijeka, Rijeka, Croatia. ${ }^{2}$ Faculty of Engineering, University of Rijeka, Rijeka, Croatia.

\section{Acknowledgements}

The paper was made with financial support of scientific project 'Mathematical and numerical modelling of compressible micropolar fluid flow' (13.14.1.3.03), University of Rijeka, Croatia.

Received: 30 December 2013 Accepted: 26 June 2014 Published online: 03 October 2014 


\section{References}

1. Eringen, AC: Simple microfluids. Int. J. Eng. Sci. 2(2), 205-217 (1964)

2. Lukaszewicz, G: Micropolar Fluids: Theory and Applications. Modeling and Simulation in Science, Engineering and Technology. Birkhäuser, Boston (1999)

3. Mujaković, N: One-dimensional flow of a compressible viscous micropolar fluid: a local existence theorem. Glas. Mat. 33, 71-91 (1998)

4. Mujaković, N: One-dimensional flow of a compressible viscous micropolar fluid: a global existence theorem. Glas. Mat. 33, 199-208 (1998)

5. Mujaković, N: One-dimensional compressible viscous micropolar fluid model: stabilization of the solution for the Cauchy problem. Bound. Value Probl. 2010, Article ID 796065 (2010)

6. Mujaković, N: Nonhomogeneous boundary value problem for one-dimensional compressible viscous micropolar fluid model: regularity of the solution. Bound. Value Probl. 2008, Article ID 189748 (2008)

7. Chen, M: Blowup criterion for viscous, compressible micropolar fluids with vacuum. Nonlinear Anal., Real World Appl. 13(2), 850-859 (2012)

8. Chen, M, Huang, B, Zhang, J: Blowup criterion for the three-dimensional equations of compressible viscous micropolar fluids with vacuum. Nonlinear Anal. TMA 79, 1-11 (2013)

9. Easwaran, C, Majumdar, S: A uniqueness theorem for compressible micropolar flows. Acta Mech. 68, 185-191 (1987)

10. Dražić, I, Mujaković, N: 3-D flow of a compressible viscous micropolar fluid with spherical symmetry: a local existence theorem. Bound. Value Probl. 2012, 69 (2012)

11. Hoff, D: Spherically symmetric solutions of the Navier-Stokes equations for compressible, isothermal flow with large, discontinuous initial data. Indiana Univ. Math. J. 41, 1225-1302 (1992)

12. Jiang, S, Zhang, P: On spherically symmetric solutions of the compressible isentropic Navier-Stokes equations. Commun. Math. Phys. 215, 559-581 (2001)

13. Fujita-Yashima, H, Benabidallah, R: Equation à symétrie sphérique d'un gaz visqueux et calorifère avec la surface libre. Ann. Mat. Pura Appl. 168, 75-117 (1995)

14. Yanagi, S: Asymptotic stability of the spherically symmetric solutions for a viscous polytropic gas in a field of external forces. Transp. Theory Stat. Phys. 29(3-5), 333-353 (2000)

15. Fujita-Yashima, H, Benabidallah, R: Unicité de la solution de equation monodimensionnelle ou a symetrie spherique d'un gaz visqueux et calorifere. Rend. Circ. Mat. Palermo XLII, 195-218 (1993)

16. Mujaković, N: Uniqueness of a solution of the Cauchy problem for one-dimensional compressible viscous micropolar fluid model. Appl. Math. E-Notes 6, 113-118 (2006)

17. Lions, JL, Dautray, R: Functional and Variational Methods. Mathematical Analysis and Numerical Methods for Science and Technology, vol. 2. Springer, Berlin (2000)

18. Lions, JL, Dautray, R: Evolution Problems I. Mathematical Analysis and Numerical Methods for Science and Technology, vol. 5. Springer, Berlin (2000)

19. Antontsev, SN, Kazhikhov, AV, Monakhov, VN: Boundary Value Problems in Mechanics of Nonhomogeneous Fluids. Studies in Mathematics and Its Applications, vol. 22. North-Holland, Amsterdam (1990)

doi:10.1186/s13661-014-0226-z

Cite this article as: Mujaković and Dražić: 3-D flow of a compressible viscous micropolar fluid with spherical symmetry: uniqueness of a generalized solution. Boundary Value Problems 2014 2014:226.

\section{Submit your manuscript to a SpringerOpen ${ }^{\circ}$ journal and benefit from:}

- Convenient online submission

- Rigorous peer review

- Immediate publication on acceptance

- Open access: articles freely available online

- High visibility within the field

- Retaining the copyright to your article 